\title{
Primary Candida guilliermondii Infection of the Knee in a Patient without Predisposing Factors
}

\author{
Gun Woo Lee, Tae-Hun Kim, and Jung-Hwan Son \\ Department of Orthopedic Surgery, Gospel Hospital, Kosin University, 34 Amnam-Dong, Seo-gu, Busan 602-702, Republic of Korea \\ Correspondence should be addressed to Jung-Hwan Son, junghson@dreamwiz.com
}

Received 23 October 2011; Accepted 6 December 2011

Academic Editor: T. Ottenhoff

Copyright () 2012 Gun Woo Lee et al. This is an open access article distributed under the Creative Commons Attribution License, which permits unrestricted use, distribution, and reproduction in any medium, provided the original work is properly cited.

Isolated primary candidal infection of joint is extremely rare, with only a few reported cases. It occurs as a result of accidental implantations of fungus during traumatic procedures, such as surgery, and is usually reported in patients with predisposing factors such as immunosuppression, malignancy, and drug abuse. If left untreated, irreversible deformity and pain with severe osteoarticular destruction occur. Thus, early diagnosis and treatment are important. This paper presents a case of 72-year-old man with primary C. guilliermondii infection of knee joint without predisposing factors and previous traumatic procedures, who was misdiagnosed with advanced degenerative osteoarthritis. Our case is the second case of primary C. guilliermondii arthritis of knee to be reported in the English-language literature and the first to be successfully treated with total knee arthroplasty following IV amphotericin B and oral fluconazole. Primary candidal infection of joint is generally asymptomatic or involves only mild pain and swelling in the affected knee. Thus, although the majority of knee joint infections are of a pyogenic or tuberculous origin, if a patient complains of mild pain and swelling in the knee and has mild signs of infection, the possibility of fungal infection should be considered.

\section{Introduction}

Candidal infection of knee joint is rare. It is primarily reported in immunosuppressed patients as a sequela of prior episodes of transient candidemia or as a result of disseminated disease [1-3]. In the majority of cases, candidal arthritis is the result of hematogenous dissemination. Primary candidal infection of joint is extremely rare, with only a few reported cases. It most often occurs as a result of accidental implantation of fungus during traumatic procedures, such as surgery, intra-articular injections, or implantation of infected joint prostheses [4-6], and also in human-immunodeficiency-virus-(HIV)-infected intravenous drug users $[7,8]$ and heroin addicts [9]. While some Candida species, including C. albicans, C. krusei, C. parapsilosis, C. tropicalis, C. glabrata, and C. lusitaniae, have been incriminated in the etiology of osteoarticular infections [10-14], C. guilliermondii joint infection has been reported only once, by Graham and Frost [15]. However, the case reported by Graham and Frost occurred in the setting of rheumatoid arthritis, which is one of the minor predisposing factors for candidal infection of joint.
Here we report a case of primary C. guilliermondii infection of the knee joint in a patient without predisposing factors that was successfully treated by total knee arthroplasty following intravenous amphotericin B and oral fluconazole. To the best of our knowledge, our case is the second case of primary C. guilliermondii infection of joint reported in the English-language literature, and the first in a patient without predisposing factors. Additionally, ours is the first reported case to be successfully treated by total knee arthroplasty following intravenous amphotericin B and oral fluconazole in case of candidal joint infection.

\section{Case Report}

A 72-year-old man presented to our clinic with a 1-year history of pain and swelling of the right knee. He had been having intermittent pain in both knees for 15 years. Medication for mild degenerative arthritis had been recommended, but the patient refused treatment. A year before he presented to our clinic, he visited another clinic due to the aggravation of pain and swelling of the right knee and was diagnosed with 


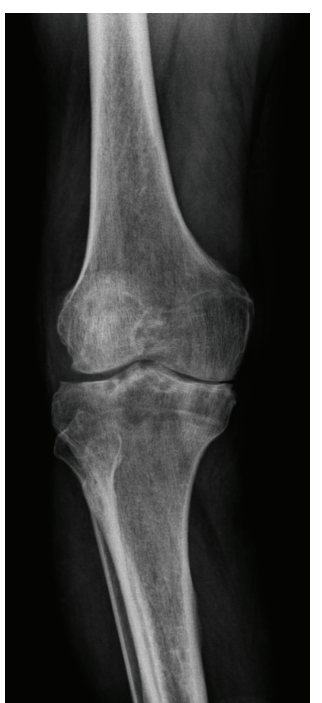

(a)

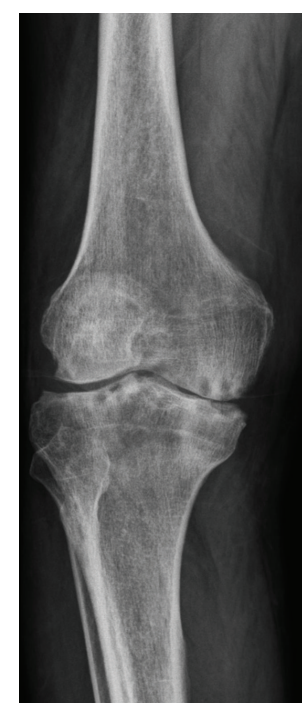

(b)
FIGURE 1: (a) Standing knee radiograph showed multiple patched subchondral lesions of the distal femur and proximal tibia, joint space narrowing, and definite osteophyte. (b) After disappearing of the infection sign, standing knee radiograph showed aggravation of intra-articular deformity.

severe degenerative arthritis by radiologic and physical exam, and a decision was made to perform total knee arthroplasty. During the operation, severe inflammation and an abscess were found inside the knee joint; therefore, bacterial culture was obtained and arthroplasty was not performed. After the operation, he was diagnosed by culture with unknownoriginated joint infection and transferred to our clinic. The medical history was negative for rheumatoid disease, cancer, renal disease, and HIV. He was a nonsmoker and nondrinker and did not take any steroids or illegal drugs. On physical examination, the patient had signs of infection, including swelling and localized warmth, of right knee. Knee radiographs revealed multiple patched subchondral lesions of the distal femur and proximal tibia, severe joint space narrowing, and definite osteophytes (Figure 1(a)). These findings were suggestive of a degenerative knee joint (K-L grade IV).

The laboratory results were as follows: white blood cell (WBC), $11,200 / \mathrm{mm}^{3}\left(4-10 \times 10^{3} / \mathrm{mm}^{3}\right)$; erythrocyte sedimentation rate (ESR), $97 \mathrm{~mm} / \mathrm{hr}(0-15 \mathrm{~mm} / \mathrm{hr})$; C-reactive protein (CRP), $19.2 \mathrm{mg} / \mathrm{dL}(0-5 \mathrm{mg} / \mathrm{dL})$. Tests for antinuclear antibody (ANA), rheumatoid factor (RF), and HLA $B_{27}$ were all negative. The joint fluid cytology findings were as follows: appearance, cloudy and reddish; WBC, 17,200/ $\mathrm{mm}^{3}$ $\left(0-200 / \mathrm{mm}^{3}\right)$ with $85 \%$ neutrophils and $10 \%$ lymphocytes. The Gram stain and acid-fast bacteria (AFB) stain showed no bacteria or tuberculosis. Candida guilliermondii was cultured in the joint fluid on hospital day 7 and was confirmed on hospital day 8 . Amphotericin B $(0.7 \mathrm{mg} / \mathrm{kg} / \mathrm{day})$ was given intravenously. Two weeks later, CRP had normalized. After 4 weeks, the amphotericin B was discontinued and oral fluconazole ( $400 \mathrm{mg} /$ day) was prescribed for 6 months. The last culture of joint fluid was negative for C. guilliermondii,

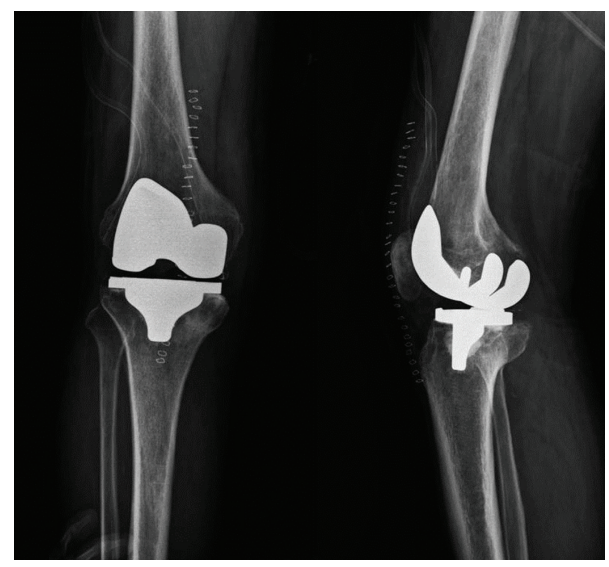

FIgure 2: Primary total knee replacement with the Duracon prosthesis (Howmedica, Rutherford, NJ, USA) was performed.

and the patient had no complaints of pain or swelling. The ESR/CRP was continuously maintained at normal state. After the inflammation had resolved, the patient had pain on the medial side of the knee joint with ambulation, and rechecking knee radiographs revealed aggravation of the patient's intra-articular deformity due to the candidal infection (Figure 1(b)). After 4 months, primary total knee replacement with a Duracon prosthesis (Howmedica, Rutherford, NJ, USA) was performed (Figure 2). The wound was daily dressed, and intravenous amphotericin was injected; however, redness and swelling were observed at the proximal operative site 1 week postoperatively (Figure 3 ).

The patient was diagnosed with a superficial infection, and wound debridement and closure were performed. So far, after discharge, there were no abnormal findings or signs of infection.

\section{Discussion}

In our patient, infection of the knee joint occurred without predisposing factors, such as autoimmune disease, HIV, chronic steroid use, cancer, or drug abuse; therefore, most clinicians suspected pyogenic or tuberculosis infection and did not consider fungal infection because it rarely occurs in normal population [16]. Only a few cases of primary candidal infection have been reported. In our knowledge, primary candidal infection has been reported in less than 15 cases, mostly in neonates [17], septicemic patients, HIV patients, and drug abusers [7-9]. While only some Candida species have been incriminated in the etiology of osteoarticular infections, C. guilliermondii has been reported only one time, by Graham and Frost [15]. Graham reported that a patient with longstanding rheumatoid arthritis and myasthenia gravis presented with mild knee pain and swelling and suggested that the unusualness organism, of the apparent chronicity of the infection, and the ultimate success of the surgical treatment are features of this organism. However, that case occurred in a patient of rheumatoid arthritis with systemic corticosteroid, which is one of predisposing factors. 


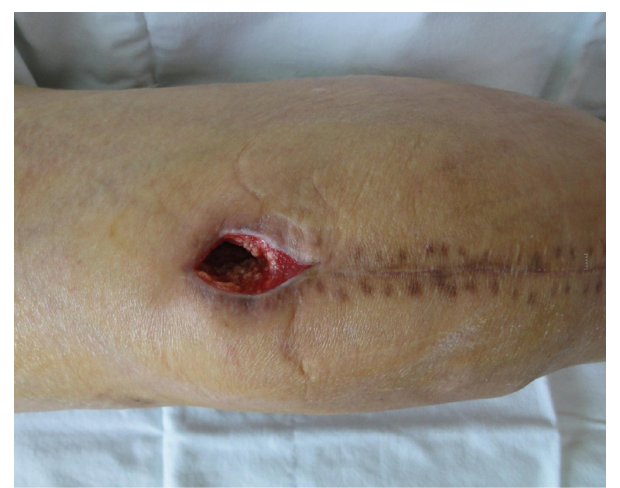

(a)

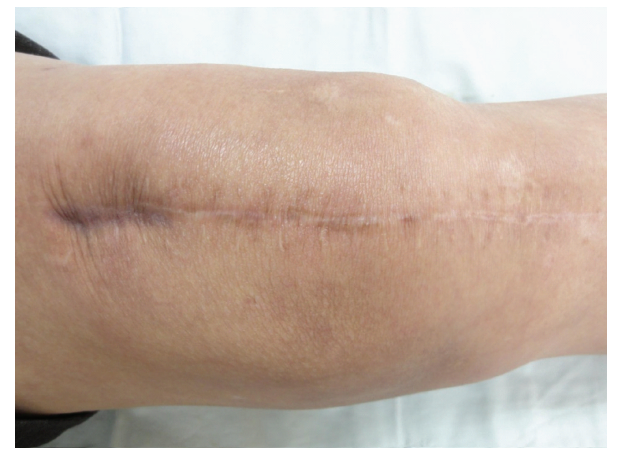

(b)

Figure 3: (a) Superficial infection at proximal operative site was noted at postoperative week 2. Debridement and closure were performed. (b) After 6 months of follow-up, there was no abnormal finding such as inflammation, and he has led a normal life.

Disseminated Candida infection manifest with systemic symptoms including cutaneous and ocular lesions, so early diagnosis and treatment are possible. However, primary candidal infection of joint usually has mild, chronic course with little pain and few articular symptoms, leading to delays in diagnosis that can be as long as several years $[14,18]$. Due to these ambiguous symptoms, primary candidal infection is often misdiagnosed as simple degenerative osteoarthritis of the knee, as in this case. However, if left untreated, irreversible deformity and pain with severe osteoarticular destruction occur. Thus, early diagnosis and treatment are important. Thus, if there are minimal infectious signs, careful physical examination and attentive analysis of blood and joint fluid should be conducted to avoid incorrect diagnosis and treatment. In addition, because the majority of knee joint infections are of a pyogenic or tuberculous origin, most physicians do not perform the study about fungus infection. Thus, if there is an ambiguous case, which shows mild pain, swelling, and infection sign, a study about fungal infection should be also done.

Definitive information on the treatment of native joint Candida infection is limited [19]. Both amphotericin B and fluconazole have been successfully used to treat infection with susceptible strains, as long as adequate drainage is provided [17]. Because successful treatment with amphotericin $\mathrm{B}$ is more commonly reported, we initiated treatment with intravenous amphotericin B, which was changed to oral fluconazole 4 weeks later. A long-term survey of Candida demonstrated that fluconazole has fewer side effects and better antifungal effects than amphotericin B [20]. Therefore, we prescribed fluconazole for 6 months. Six months later, neither signs of inflammation nor side effects were observed.

As determined by a search of Medline for articles between 1966 and January 2011, our case is the second case of primary C. guilliermondii infection of knee joint to be reported in the English-language literature and the first to be successfully treated with total knee arthroplasty following IV amphotericin B and oral fluconazole. Primary candidal infection of joint is generally asymptomatic or involves only mild pain and swelling in the affected knee. Thus, if a patient complains of mild pain and swelling in the knee and has mild signs of infection, the possibility of fungal infection should be considered.

\section{Conclusion}

Primary candidal infection of joint is extremely rare, with only a few reported cases. This infection is generally asymptomatic or involves only mild pain and swelling in the affected knee. However, if left untreated, irreversible deformity and pain with severe osteoarticular destruction occur. Thus, early diagnosis and treatment are important. Although the majority of knee joint infections are of pyogenic or tuberculous origin, if a patient complains of mild pain and swelling in the knee and has mild signs of infection, the possibility of fungal infection should be considered.

\section{Disclosure}

The authors did not have any relevant financial disclosure or personal relationships.

\section{Acknowledgment}

The authors thank Chung-Kue Kim, MD, for technical assistance.

\section{References}

[1] V. J. Fraser, M. Jones, J. Dunkel, S. Storfer, G. Medoff, and W. C. Dunagan, "Candidemia in a tertiary care hospital: epidemiology, risk factors, and predictors of mortality," Clinical Infectious Diseases, vol. 15, no. 3, pp. 414-421, 1992.

[2] B. Turgut, O. Vural, M. Demir, and M. Kaldir, "Candida arthritis in a patient with chronic myelogenous leukemia (CML) in blastic transformation, unresponsive to fluconazole, but treated effectively with liposomal amphotericin B," Annals of Hematology, vol. 81, no. 9, pp. 529-531, 2002.

[3] W. B. Hsieh and C. Leung, "Candidal arthritis after complete treatment of systemic candidiasis," Journal of the Chinese Medical Association, vol. 68, no. 4, pp. 191-194, 2005.

[4] H. J. Arnold, A. Dini, G. Jonas, and E. L. Zorn, "Candida albicans arthritis in a healthy adult," Southern Medical Journal, vol. 74, no. 1, pp. 84-85, 1981.

[5] V. Kak and P. H. Chandrasekar, "Bone and joint infections in injection drug users," Infectious Disease Clinics of North America, vol. 16, no. 3, pp. 681-695, 2002. 
[6] C. H. Lee, J. M. Oh, S. R. Oh, M. Yoo, and M. S. Lee, "Candida arthritis after arthroscopic arthroplasty in a patient without predisposing factors," Open Rheumatology Journal, vol. 4, pp. 7-9, 2010.

[7] H. Edelstein and R. McCabe, "Candida albicans septic arthritis and osteomyelitis of the sternoclavicular joint in a patient with human immunodeficiency virus infection," Journal of Rheumatology, vol. 18, no. 1, pp. 110-111, 1991.

[8] S. M. Fernandez, A. Cardenal, A. Balsa et al., "Rheumatic manifestations in 556 patients with human immunodeficiency virus infection," Seminars in Arthritis and Rheumatism, vol. 21, no. 1, pp. 30-39, 1991.

[9] J. Bisbe, J. M. Miro, X. Latorre et al., "Disseminated candidiasis in addicts who use brown heroin: report of 83 cases and review," Clinical Infectious Diseases, vol. 15, no. 6, pp. 910-923, 1992.

[10] J. S. Goodman, D. G. Seibert, G. E. Reahl Jr., and R. W. Geckler, "Fungal infection of prosthetic joints: a report of two cases," Journal of Rheumatology, vol. 10, no. 3, pp. 494-495, 1983.

[11] D. R. Mandel, A. M. Segal, A. J. Wysenbeek, and L. H. Calabrese, "Two unusual strains of Candida arthritis," American Journal of the Medical Sciences, vol. 288, no. 1, pp. 25-27, 1984.

[12] L. de Clerck, J. Dequeker, R. Westhovens, and D. Hauglustaine, "Candida parapsilosis in a patient receiving chronic hemodialysis," Journal of Rheumatology, vol. 15, no. 2, pp. 372-374, 1988.

[13] V. Q. Nguyen and R. L. Penn, "Candida krusei infectious arthritis. A rare complication of neutropenia," American Journal of Medicine, vol. 83, no. 5, pp. 963-965, 1987.

[14] D. Katzenstein, "Isolated Candida arthritis: report of a case and definition of a distinct clinical syndrome," Arthritis and Rheumatism, vol. 28, no. 12, pp. 1421-1424, 1985.

[15] D. R. Graham and H. M. Frost, "Candida guilliermondi infection of the knee complicating rheumatoid arthritis: a case report," Arthritis and Rheumatism, vol. 16, no. 2, pp. 272-277, 1973.

[16] X. Zhao, S. Chen, A. Deanda Jr., and J. Kiev, "A rare presentation of tuberculosis," American Surgeon, vol. 72, no. 1, pp. 9697, 2006.

[17] R. Kohli and S. Hadley, "Fungal arthritis and osteomyelitis," Infectious Disease Clinics of North America, vol. 19, no. 4, pp. 831-851, 2005.

[18] R. S. Pastan, S. L. Silverman, and D. L. Goldenberg, "A musculoskeletal syndrome in intravenous heroin users. Association with brown heroin," Annals of Internal Medicine, vol. 87, no. 1, pp. 22-29, 1977.

[19] J. Paul, C. Kirchhoff, A. B. Imhoff, and S. Hinterwimmer, "Infection after arthroscopy," Der Orthopäde, vol. 37, no. 11, pp. 1048, 1050-1052, 1054-1055, 2008.

[20] D. L. Horn, D. Neofytos, E. J. Anaissie et al., "Epidemiology and outcomes of candidemia in 2019 patients: data from the prospective antifungal therapy alliance registry," Clinical Infectious Diseases, vol. 48, no. 12, pp. 1695-1703, 2009. 


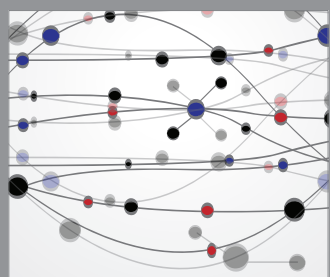

The Scientific World Journal
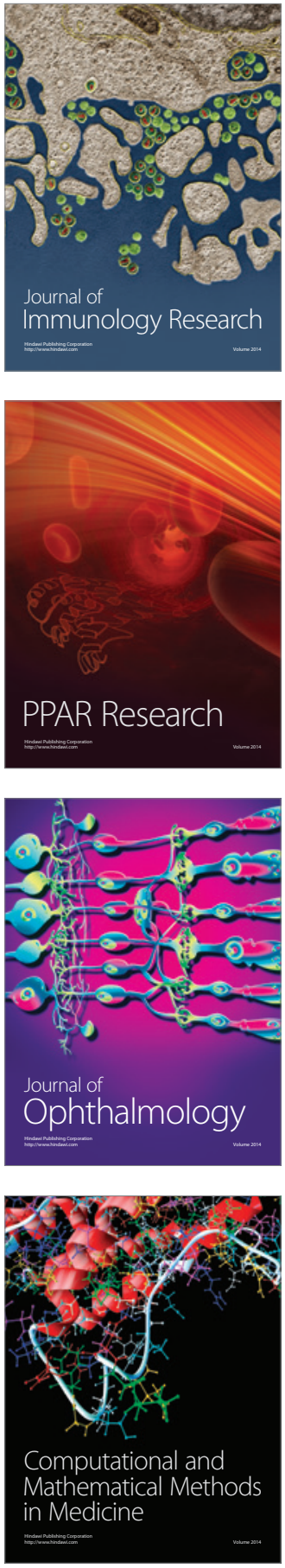

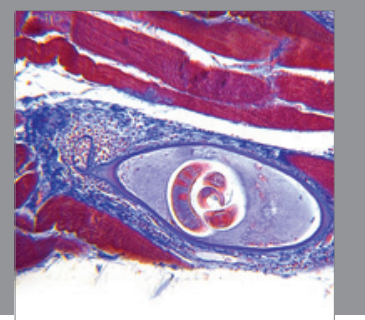

Gastroenterology

Research and Practice
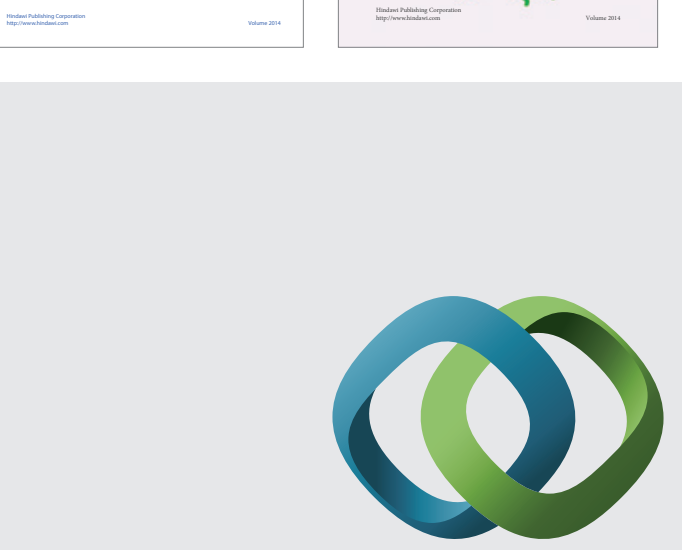

\section{Hindawi}

Submit your manuscripts at

http://www.hindawi.com
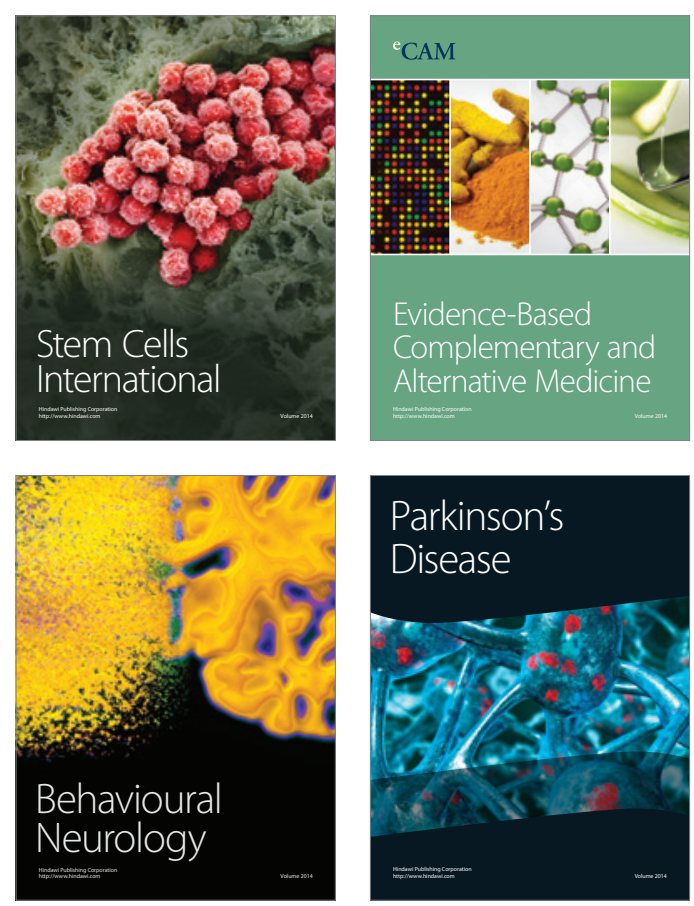

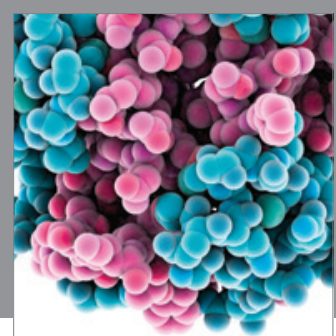

Journal of
Diabetes Research

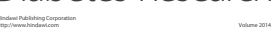

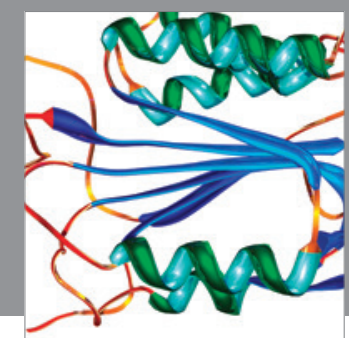

Disease Markers
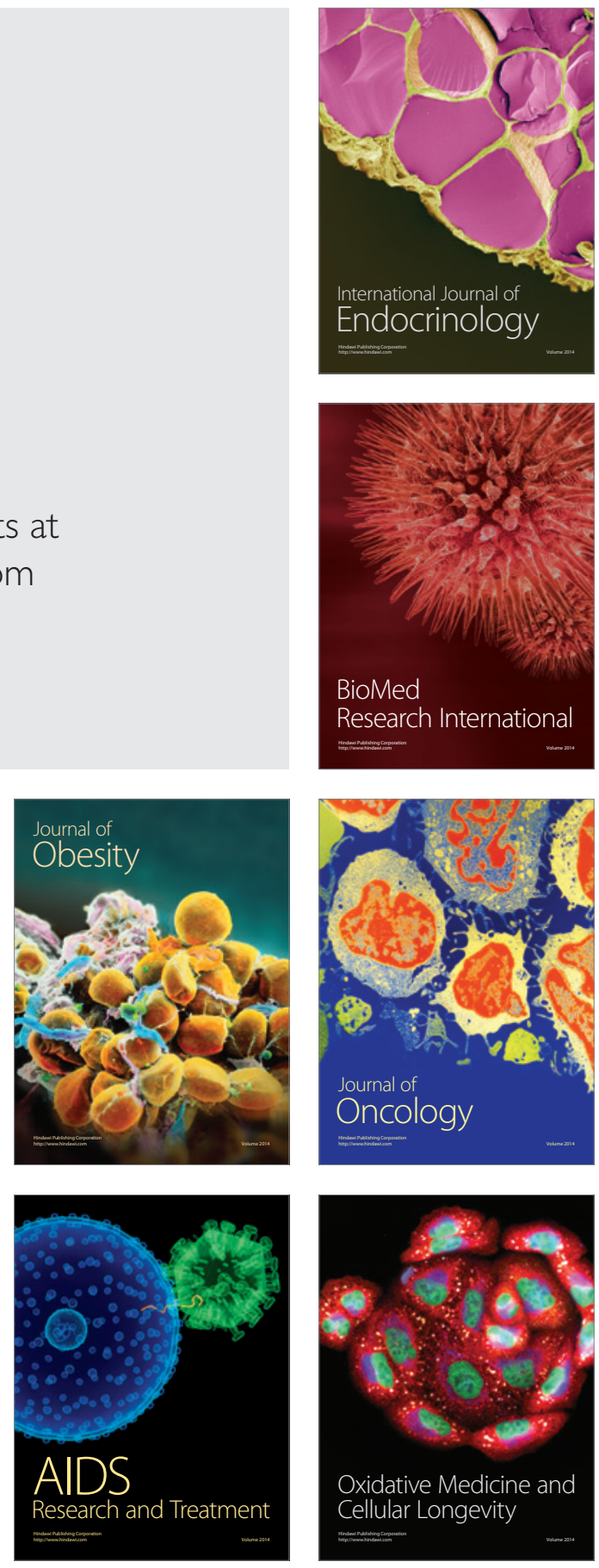